\title{
Erratum: Bouncing motion and penetration dynamics in multicomponent Bose-Einstein condensates [Phys. Rev. A 93, 033615 (2016)]
}

\author{
Yujiro Eto, Masahiro Takahashi, Keita Nabeta, Ryotaro Okada, Masaya Kunimi, Hiroki Saito, and Takuya Hirano \\ (Received 29 November 2017; published 13 December 2017)
}

DOI: 10.1103/PhysRevA.96.069904

The values of the interspecies scattering length $a_{1,2}$ and the degree of miscibility $\Delta_{a}$ between $|1,-1\rangle$ and $|1,0\rangle$ in Table I and Fig. 2 are incorrect. The correct values should be $a_{1,2}=100.40 a_{0}[1,2]$ and $\Delta_{a}=-0.0023$. Due to this correction, the ground state for $|1,-1\rangle$ and $|1,0\rangle$ is changed from weakly immiscible to weakly miscible. Even though this change is made, our claim "observation of the mutual penetration for immiscible condensates" remains unaltered since the mutual penetration for immiscible condensates is observed in the three-component system as shown in Fig. 6 and numerically confirmed as shown in Fig. 4. Therefore, the corrections do not affect the conclusions of the paper.

[1] E. G. M. van Kempen, S. J. J. M. F. Kokkelmans, D. J. Heinzen, and B. J. Verhaar, Interisotope Determination of Ultracold Rubidium Interactions from Three High-Precision Experiments, Phys. Rev. Lett. 88, 093201 (2002).

[2] A. Widera, F. Gerbier, S. Fölling, T. Gericke, O. Mandel, and I. Bloch, Precision measurement of spin-dependent interaction strengths for spin-1 and spin- $2{ }^{87} \mathrm{Rb}$ atoms, New J. Phys. 8, 152 (2006). 